\title{
Application Research on Uncertainty Inference Based on Evidence Theory
}

\author{
Li Yang ${ }^{1, a}$ \\ ${ }^{1}$ Nanchang Institute of Science \& Technology, Nanchang, 330108, China \\ a191815045@qq.com
}

Keywords: uncertainty inference; evidence theory; certainty factor

\begin{abstract}
The modeling and reasoning with uncertain context information is one of key problems of building context-aware applications in pervasive computing environment. In this paper, a method of modeling uncertain contexts is proposed by combining the DS theory with ontology model. Two application cases of uncertainty inference based on evidence theory are provided, and the feasibility and effectiveness of the proposed method are verified.
\end{abstract}

\section{Introduction}

Evidence theory is a common method to deal with uncertain reasoning is incomplete, unclear, uncertain, as with other uncertainty reasoning: Bayesian inference, fuzzy logic reasoning and rule reasoning based on compared with measurements in combination with the advantages [1]. Evidence theory can combine the advantages of other methods while improving its shortcomings. It extends from probability to fuzzy set. It can not only combine Bias's reasoning with prior information, but also handle fuzzy concept evidence. The language in the application, the evidence theory is used in different levels and excellent performance, for example: index system, fault diagnosis, expert system, controller modeling, artificial intelligence and decision analysis, with the theory of evidence in the continuous promotion of various fields and should be used, the theory also further enhance. Evidence theory has become an important method of uncertainty reasoning. Many corresponding literatures have been published both at home and abroad. This paper will introduce some common evidence theory research and application to provide references for readers to further study.

\section{Summary of Evidence Theory}

Dempster first proposed the theory of evidence in the study of statistical problem in 1967 [2]. He gave the concept of upper and lower probabilities and their synthetic rules. For the first time, we explicitly gave the probability of not satisfying additivity. Schaefer generalized it to more general cases and made it systematized and theorized.

Suppose there is a possibility, we use non-empty set to represent all the possible results for this problem, Schaefer pointed out that the possibility of results is assumed to be independent of each other, mutual exclusion, but the possibility of a perfect interpretation. Its choice comes from our prior knowledge. It comes from our cognitive structure. It comes from what we want to know and what we know about theta subset is called a proposition. Theta power set that all possible propositions set by both the collection of all subsets of theta composition, recognition framework is the foundation of the theory of evidence, all the concepts and functions of evidence theory are based on the recognition framework, evidence combination rules are based on the same recognition framework, any A belongs to 0 , then $\mathrm{m}(\mathrm{A})$ also known as the basic probability assignment proposition $\mathrm{A}, \mathrm{m}(\mathrm{A})$ that is assigned to a confidence measure of $A$ itself, which support the proposition itself A degree, and does not support A subsets. M (A) is also known as a hypothetical mass function or a mass function. The determination of the mass function is given by the experience of the people or based on the data of the sensor.

The necessary condition for the theory of evidence is that the evidence of the merger must be 
independent, but in many cases, it is not a reasonable assumption. Has used two methods for solving mathematician element probability and evidence theory is an example, different results. The former is in conformity with the reality, and the latter violates the objective fact. In a multi intelligent sensor system, the information possessed by each sensor is always related to its environment. Even if the information it represents has different descriptions of the time and space for the environment, the confidence level is different. Therefore, in information fusion, we must change it so that it can be adapted to the non-independent evidence [3].

\section{Cases of Uncertainty Inference Application Based on Evidence Theory}

\subsection{Case One}

The theory of evidence belongs to the category of artificial intelligence, which is first applied to the expert system and can deal with uncertain information. As an uncertain reasoning method, the main feature of evidence theory is that it satisfies weaker conditions than Bayesian probability theory, and can express "uncertain" and "do not know" directly [4].

After that, many technologies have improved and developed the DS theory, one of which is the Evidential reasoning algorithm. The ER algorithm is developed based on the confidence evaluation framework and the DS theory. ER algorithm has been successfully applied to vehicle evaluation and analysis, cargo ship design, naval system safety analysis and synthesis, software system safety performance analysis, modification of ferry design and administrative vehicle evaluation organization evaluation.

In many application fields of medical diagnosis, target recognition, military command, need to consider from multiple sources of uncertain information, such as information, many experts from multiple sensors opinions and so on, to solve the problem, play an important role in combination rules of evidence theory in this aspect of the solution.

If a friend or foe (IFF Identification, Friend aircraft identification or (Foe) sensor. Aircraft recognition (IFF, Identification Friend or Foe) sensor has also been referred to as' aircraft identification device), from a transponder aircraft received a response. If an aircraft transponder, so it should be carried out immediately by the echo response identification code. If the aircraft receiving the response does not receive the response of an aircraft $A$, the default processing result of the received response is that the aircraft $A$ is an enemy aircraft. A plane $A *$ may be due to the following reasons: failed to send response information (1) failed. Aircraft identification $A^{*}$; (2) the fault transponder $A^{*}$; (3) have no enemies' aircraft identifier $A^{*}$; (4) an aircraft identifier $A^{*}$ is disturbed $\left(5 ; A^{*}\right)$ received to keep its radar silent.

Because of the fault plane assumed foe recognition, leading to a target plane has a 0.7 chance of being the evidence, which is the only bomber and fighter aircraft. Thus, this assignment is M1 Mass $(\{B, F\})=0.7$, which means the M1 provided by the first aircraft. Recognition evidence Mass value.

The rest of the trust will be left to ourselves as an expression of opinion environment, part of the M1 $(\{1-0.7\}=$ ourselves $)=0.3$. Pay attention to 'unexpressed opinion' is neither trust nor distrust. Probability are given different results: $\mathrm{P}$ (enemy) $=0.7$.

Each Mass can be formalized into a function, which maps every element in a power set to a real number of interval $[0,1]$. The formalization of the function is described as. In practice, the Mass collection space is usually defined as 0 (zero), $\mathrm{m}$ (net) $=0$. All subsets are Mass and 1 . For example, in an aircraft environment, when new evidence is available, we want to combine all the evidence to produce a better trust evaluation. To illustrate how to combine evidence (also known as a combination of evidence), we first look at a special case of a general formula of the combination of evidence.

It is assumed that another type of sensor identifies the target aircraft as a bomber with 0.9 trust. Now, from the sensor evidence Mass: $\mathrm{M} 1(\{\mathrm{~B}, \mathrm{~F}\})=0.7, \mathrm{M} 1=0.3$ (ourselves); $\mathrm{M} 2(\{\mathrm{~B}\})=0.9, \mathrm{M} 2$ $=0.1$ (ourselves). Among them, M1 and M2 correspond to the first and second types of sensors. A special form of Dempster's rule of combination using the combination to produce Mass, $\mathrm{X}$ and $\mathrm{Y}$ all 
elements of the establishment of the operator and orthogonal or direct and.

The Dempster rules of combination of two Mass to produce a new Mass, the new Mass said the initial consensus may be conflict among evidences. The new Mass expresses the common evidence element by gathering consensus only on the intersection of Mass, and the intersection of the sets. It is very important that the evidence for combination must be an independent error (independent errors). Note that the evidence independent evidence collected by independent errors. We give the Dempster rules of combination, which after each intersection will follow a numerical.

\subsection{Case Two}

D-S evidence theory has a fatal error, and its reasoning results are unbelievable. Due to the above allocation of mass, the relationship between the elements that identify the power set of the framework becomes very unique. The mistake in understanding this unique relationship is the root of all the problems in the theory of evidence.

It must be realized that from the point of view of the mass, the elements that identify the power set of the framework are independent of each other! For example, for a proof, $m(A), m(B)$ and $m(A, B)$ is the Never mind. Unlike in probability theory that $\mathrm{P}(\mathrm{A}, \mathrm{B})=\mathrm{P}(\mathrm{A})+\mathrm{P}(\mathrm{B})$ (note to the identification of the elements in the framework are generally independent of each other) this relationship. In other words, though the mass assignment is carried out on the discernment frame power set, once the mass is allocated, the elements in the power set, that is, the subset of the identification frameworks, is no longer a common set. Therefore, there is no basic meaning and nature of the common set. As a common set, the nature of the evidence in theory are no longer applicable. Or, to be more exact, the theory of evidence should just "borrow" some of the symbols in the set theory. In evidence theory, A, B only for the part of A and B cannot be separated, but not can be separated from that part of the A and B. The theory of evidence is the confusion between the "borrowing" and "real use", which leads to the error.

Because of such a fundamental mistake, evidence reasoning often leads to some weird reasoning. For many years, it is believed that the evidence theory is prone to this strange phenomenon when the conflict of evidence is serious. Therefore, people have studied various ways to amend the evidence reasoning when the conflict is serious. Now it seems that the basis of evidence reasoning is problematic, but the problem is more serious when the conflict is serious. Such a theory cannot solve the problem by "revising". He points out that DS evidence theory has only some value in one problem, that is, the description of information which is not ideal, that is, mass assignment is used to solve the description problem of information such as "unclear" and "do not know". In addition, the core content of the theory of evidence, including the formation of confidence interval and the synthesis of evidence, is unbelievable.

To solve the problem of reasoning with "indistinct" and "unknown" information. Document puts forward a concise solution. It is to return the problem proposed by the theory of evidence back to the framework of probability reasoning. The method is, if we can get the mass assignment, we can divide the mass of each compound element equally among all basic elements, and each basic element will take the mass summation of this method as a certain probability. After the probability, it can be deduced by means of probabilistic reasoning.

Fuzzy reasoning is an uncertain reasoning technology based on the fuzzy set theory proposed by the American scholar Zadeh. Its specialty is the reasoning problem of dealing with uncertain information such as intension or extension of "not clear". This kind of fuzzy information is common in the real problem, so the fuzzy reasoning has a wide application space. Fuzzy reasoning is the basic framework of the classical deductive reasoning, which imitates the handling of fuzzy information by human thinking. Like deductive reasoning, fuzzy reasoning can also be expressed as a syllogism, which is a big premise, a small premise and a conclusion. The difference is that the concepts involved in deductive reasoning must be abstract, clear and two senses. The inference rules used are the correct theorems in a certain theoretical framework, and the conclusions obtained are reliable. This rigor and reliability are the greatest advantages of deductive reasoning. The rigor of form and logic makes deductive reasoning particularly easy to be realized by machine. However, the problem of fuzzy 
reasoning generally does not have the condition of deductive reasoning. These questions either contain unclear concepts or have no absolute axiomatic system as a basis for reasoning. Deductive reasoning on such a problem and a reasoning machine based on it are powerless. But in this case, people can still think and reason. The basic idea of fuzzy reasoning is to use the fuzzy set method to simulate the process of human thinking and reasoning. One of the important features of fuzzy reasoning is that the large premise is not completely consistent with the small premise.

Combined with expert experience rule base and uncertainty reasoning method based on DS theory, the interference cause and monitoring scheme of the civil aviation radio interference expert system is relatively accurate and efficient. Even if the experience knowledge in the rule base is incomplete, it can remind the radio interference to provide more information to the investigating staff, and assist the interference investigation with other auxiliary means. The accuracy of the civil aviation radio interference investigation expert system "is mainly reflected in the consistency with the literature search results, but because the rules are not perfect, interference reasons the system failed to give literature; efficiency is mainly reflected in the literature staff before determining the true causes of interference, spent more time monitoring, data analysis, which cannot be a one-time to accurately determine the causes of interference. Through the "interference expert system of civil aviation radio interference", input the characterization of interference provided by the interfering units, it can give the possible cause of interference immediately, and get the most probable interference cause according to the determining factors of the interference characterization, and greatly shorten the monitoring time. Experiments and practices show that the system can better help users investigate and deal with interference. At the same time, the knowledge of the system is given in the form of uncertainty knowledge, which is more consistent with the actual situation, and the user interface is friendly, which is easy for users to understand and use.

\section{Conclusion}

Evidence theory is a method of uncertainty reasoning which is expressed by confidence function. It uses the confidence function to restrict the events that are difficult to get precise probability. However, when the constraint condition becomes a strict probability, it can be converted into a probability theory. The theory of evidence can deal with uncertainty caused by randomness and uncertainty caused by fuzziness.

\section{References}

[1] Jin Liuqian, Xu Yanga. Method for uncertain multi-attribute decision making based on evidential reasoning and third-generation prospect theory [J]. Control and Decision, 2016, 31(1): 105-113.

[2] Jin Liuqian, Xu Yang, Fang Xin, et al. Uncertainty inference method based on Dempster-Shafer evidence theory and its application. Computer Engineering and Applications [J]. Computer Engineering and Applications, 2015, 51(10): 6-11.

[3] Li Yanna, Qiao Xiuquan, Li Xiaofeng. An Uncertain Context Ontology Modeling and Reasoning Approach Based on D-S Theory [J]. Journal of Electronics \& Information Technology, 2010, 32(8): 1806-1811.

[4] Zhou Dimin, Duan Guoyun. Research on application of evidence theory in uncertainty reasoning [J]. Application Research of Computers, 2010, 27(12): 4433-4435. 